\title{
UNCOVERING THE DEEPLY EMBEDDED ACTIVE GALACTIC NUCLEUS ACTIVITY IN THE NUCLEAR REGIONS OF THE INTERACTING GALAXY Arp 299
}

\author{
A. Alonso-Herrero ${ }^{1,14}$, P. F. Roche ${ }^{2}$, P. Esquej ${ }^{3}$, O. González-Martín ${ }^{4,5}$, M. Pereira-Santaella ${ }^{6}$, C. Ramos Almeida ${ }^{4,5}$, \\ N. A. Levenson ${ }^{7}$, C. Packham ${ }^{8}$, A. Asensio Ramos ${ }^{4,5}$, R. E. Mason ${ }^{9}$, J. M. Rodríguez Espinosa ${ }^{4,5}$, C. Alvarez $^{4,5}$, \\ L. Colina ${ }^{3}$, I. Aretxaga ${ }^{10}$, T. Díaz-Santos ${ }^{11}$, E. Perlman ${ }^{12}$, and C. M. Telesco $^{13}$ \\ ${ }^{1}$ Instituto de Física de Cantabria, CSIC-UC, E-39005 Santander, Spain; aalonso@ifca.unican.es \\ ${ }^{2}$ Astrophysics Department, University of Oxford, Oxford OX1 3RH, UK \\ ${ }^{3}$ Centro de Astrobiología, CSIC-INTA, E-28035 Madrid, Spain \\ ${ }^{4}$ Instituto de Astrofísica de Canarias, E-38205 La Laguna, Spain \\ ${ }^{5}$ Universidad de la Laguna, E-38205 La Laguna, Spain \\ ${ }^{6}$ Istituto di Astrofisica e Planetologia Spaziali, INAF, I-00133 Rome, Italy \\ ${ }^{7}$ Gemini Observatory, La Serena, Chile \\ ${ }^{8}$ University of Texas at San Antonio, San Antonio, TX 78249, USA \\ ${ }^{9}$ Gemini Observatory, Hilo, HI 96720, USA \\ ${ }^{10}$ INAOE, 72000 Puebla, Mexico \\ ${ }^{11}$ Spitzer Science Center, Caltech, Pasadena, CA 91125, USA \\ 12 Florida Institute of Technology, Melbourne, FL 32901, USA \\ ${ }^{13}$ Department of Astronomy, University of Florida, Gainesville, FL 32611, USA \\ Received 2013 August 30; accepted 2013 November 13; published 2013 November 26
}

\begin{abstract}
We present mid-infrared (MIR) 8-13 $\mu \mathrm{m}$ spectroscopy of the nuclear regions of the interacting galaxy Arp 299 (IC 694+NGC 3690) obtained with CanariCam (CC) on the $10.4 \mathrm{~m}$ Gran Telescopio Canarias (GTC). The high angular resolution $\left(\sim 0.3-0^{\prime} \cdot 6\right)$ of the data allows us to probe nuclear physical scales between 60 and 120 pc, which is a factor of 10 improvement over previous MIR spectroscopic observations of this system. The GTC/CC spectroscopy displays evidence of deeply embedded active galactic nucleus (AGN) activity in both nuclei. The GTC/CC nuclear spectrum of NGC 3690/Arp 299-B1 can be explained as emission from AGN-heated dust in a clumpy torus with both a high covering factor and high extinction along the line of sight. The estimated bolometric luminosity of the AGN in NGC 3690 is $3.2 \pm 0.6 \times 10^{44} \mathrm{erg} \mathrm{s}^{-1}$. The nuclear GTC/CC spectrum of IC 694/Arp 299-A shows $11.3 \mu \mathrm{m}$ polycyclic aromatic hydrocarbon emission stemming from a deeply embedded $\left(A_{V} \sim 24 \mathrm{mag}\right)$ region of less than $120 \mathrm{pc}$ in size. There is also a continuum-emitting dust component. If associated with the putative AGN in IC 694, we estimate that it would be approximately five times less luminous than the AGN in NGC 3690. The presence of dual AGN activity makes Arp 299 a good example to study such phenomena in the early coalescence phase of interacting galaxies.
\end{abstract}

Key words: galaxies: individual (Arp 299, IC 694, NGC 3690) - galaxies: nuclei - galaxies: Seyfert infrared: galaxies

Online-only material: color figures

\section{INTRODUCTION}

The interacting galaxy Arp 299 (Mrk 171, IC 694+NGC 3690) was identified as a mid-infrared (MIR) luminous source (Rieke \& Low 1972) more than $40 \mathrm{yr}$ ago. Subsequently, Gehrz et al. (1983) detected two bright $10 \mu \mathrm{m}$ sources coincident with the galaxy nuclei (see also Telesco et al. 1985), referred to as Arp 299-A or the nucleus of the eastern component (IC 694) and Arp 299-B or the nucleus of the western component (NGC 3690). Source B is further resolved into B1, the MIRbright nucleus, and B2, the optical-bright source (see Figure 1). Two other bright MIR sources are in the system overlapping region: Arp 299-C and Arp 299-C'. The infrared (IR) luminosity of the system is $L_{\mathrm{IR}}=6.7 \times 10^{11} L_{\odot}$ (for a distance $D=44 \mathrm{Mpc}$ ), which puts it in the luminous IR galaxy (LIRG) category.

Most of the Arp 299 IR luminosity arises from intense star formation (SF) activity across the two galaxies (see Gehrz et al. 1983; Charmandaris et al. 2002; Alonso-Herrero et al. 2000, 2009, (AAH00 and AAH09 hereafter)). However, there is also

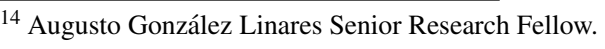

evidence of obscured active galactic nucleus (AGN) activity in the galaxy nuclei.

Based on hard X-ray observations, the nucleus of NGC 3690/Arp 299-B1 was long suspected to have a Comptonthick AGN (see, e.g., Ballo et al. 2004; González-Martín et al. 2009; Pereira-Santaella et al. 2011). Moreover, there is a hot dust continuum associated with an obscured AGN that contributes substantially to the nuclear IR emission (Gallais et al. 2004; AAH00; AAH09). Although initially thought not to be a Seyfert galaxy, García-Marín et al. (2006) measured Seyfert-like optical line ratios for Arp 299-B1.

The case for the presence of an AGN in the nuclear region of IC 694/Arp 299-A is not as clear. Apart from the tentative X-ray evidence (Ballo et al. 2004), perhaps the most convincing argument is the very long baseline interferometry identification of a flat spectrum compact radio source (Pérez-Torres et al. 2010) among the population of radio sources detected in the nuclear region of this galaxy (Neff et al. 2004).

In this Letter we present 8-13 $\mu \mathrm{m}$ spectroscopy of the two nuclei of Arp 299 obtained with CanariCam (CC; Telesco et al. 2003) on the $10.4 \mathrm{~m}$ Gran Telescopio Canarias (GTC). The GTC/CC high angular resolution 0'.3-0.'6 ( $\sim 60-120$ pe for 

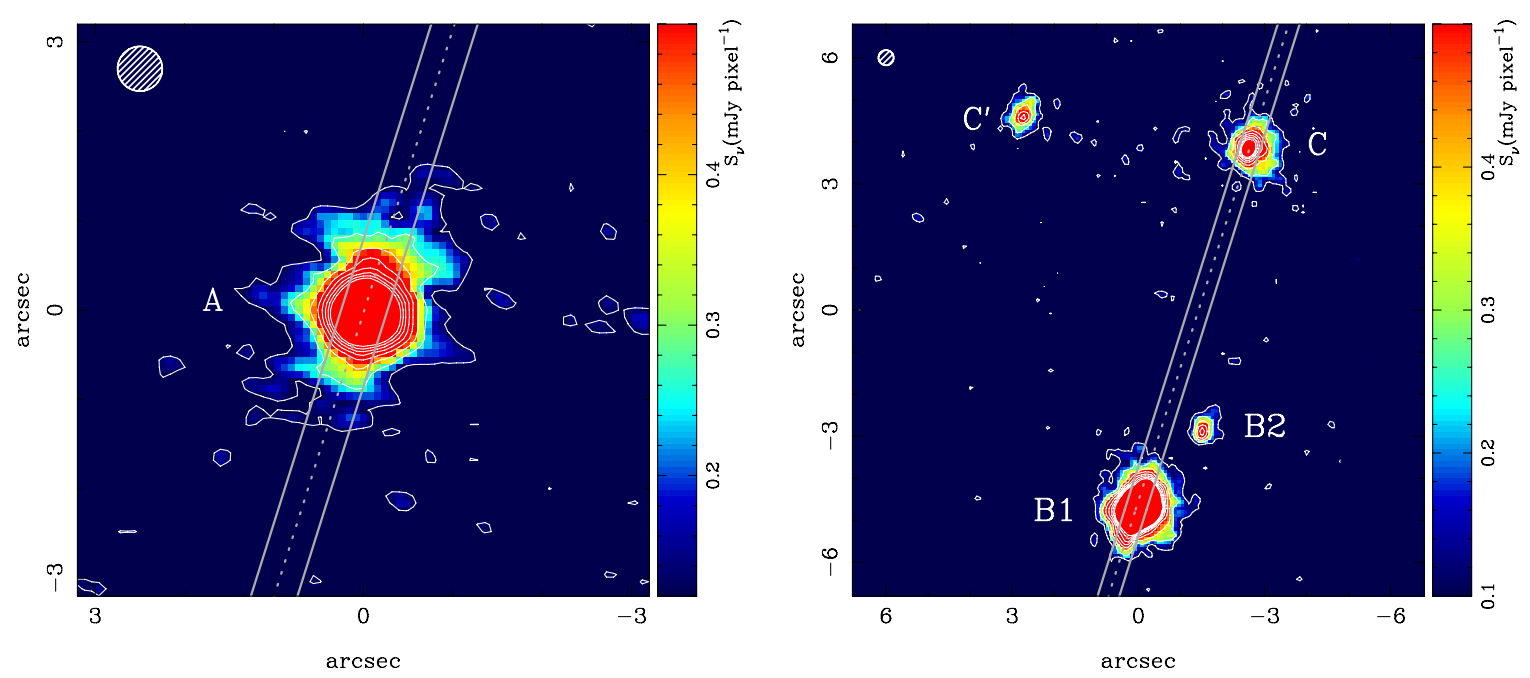

Figure 1. GTC/CC $8.7 \mu \mathrm{m}$ acquisition images of IC 694 (left panel) and NGC 3690 (right panel) shown on a linear flux scale. The projected separation between the galaxy nuclei IC 694/Arp 299-A and NGC 3690/Arp 299-B1 is approximately $21^{\prime \prime}$ or $4.5 \mathrm{kpc}$, as can be seen from Figure 5 of AAH09. We also label other IR-emitting sources in NGC 3690: B2, C, and C'. The lines indicate the location, orientation, and width of the slits. The images have been smoothed with a Gaussian function with $\sigma=0.9$ pixels. The hatched circles at the top left of the panels represent the approximate resolution of the images (FWHM). Orientation is north up, east to the left.

(A color version of this figure is available in the online journal.)

Arp 299 distance) is approximately a factor of 10 improvement over previous MIR spectroscopy of this system (Dudley 1999; Gallais et al. 2004; AAH09). We use the GTC/CC spectroscopy to shed light on the processes giving rise to the deeply nuclear embedded emission in Arp 299-A and Arp 299-B1.

\section{CANARICAM OBSERVATIONS AND DATA REDUCTION}

We obtained mid-IR high angular resolution long-slit spectroscopy of the nuclear regions of Arp 299 using CC on the GTC. The observations are part of our GTC/CC AGN guaranteed time program (PI: C. Telesco). We will use these observations together with an ESO/GTC large program (PI: A. Alonso-Herrero) to conduct a MIR survey of approximately 100 local AGN. We used the low spectral resolution $10 \mu \mathrm{m}$ grating, which covers the $N$-band $\sim 7.5-13 \mu \mathrm{m}$ with nominal $R=\lambda / \Delta \lambda \sim 175$. A 0.52 wide slit was oriented at $345^{\circ}$ so that Arp 299-B1 and C could be observed simultaneously (see Figure 1, right panel). The plate scale of the CC $320 \times 240$ Si:As detector is $00^{\prime} 08$ pixel $^{-1}$, which provides a field of view in imaging mode of $26^{\prime \prime} \times 19^{\prime \prime}$.

The observations were taken in queue mode under photometric conditions using the standard MIR chop-nod technique on 2013 January 29 and June 25 for Arp 299-A and Arp 299-B1+C, respectively. First, we obtained an acquisition image (Figure 1) through the Si-2 filter $\left(\lambda_{\mathrm{c}}=8.7 \mu \mathrm{m}\right.$ and $\Delta \lambda_{\text {cut }}=1.1 \mu \mathrm{m}, 50 \%$ cut-on/off) to ensure optimal placement of the slit. The onsource integration times for the spectroscopy were $1011 \mathrm{~s}$ for Arp 299-A and 354 s for Arp 299-B1+C. We also observed standard stars in imaging and spectroscopic mode to provide the photometric calibration, telluric correction, and slit loss correction. We used the standard stars and galaxy nuclei to measure an angular resolution at $8.7 \mu \mathrm{m}$. of 0..5-0.'6 and 0.'3 (FWHM) for Arp 299-A and Arp 299-B1+C, respectively.

We reduced the data using the $\mathrm{CC}$ pipeline REDCAN (González-Martín et al. 2013) that includes stacking of the individual observations, wavelength calibration, trace determination, spectral extraction, and flux calibration. The core emission of the two nuclei appears unresolved at the resolution of the
GTC/CC acquisition images. We extracted the one-dimensional nuclear spectra in an optimal way for a point source with the aperture size increasing with wavelength to include all the source flux. Finally, we used the observations of the standard stars to correct for slit losses. We estimated a $20 \%$ total uncertainty of the GTC/CC spectra due to flux calibration and correction for the point source extraction.

To compare with the large scale emission $(\sim 1 \mathrm{kpc})$, we used spectral mapping observations obtained with the Infrared Spectrograph (IRS; Houck et al. 2004) on board Spitzer (see AAH09 for full details about the observations). We used CUBISM (Smith et al. 2007) to extract $3 \times 3$ pixel $\left(5^{\prime \prime} .5 \times 5^{\prime \prime} .5\right)$ short-low SL1 $(\sim 7-15 \mu \mathrm{m})$ spectra. We corrected the spectra for point source emission as in Pereira-Santaella et al. (2010). Figure 2 shows the GTC/CC nuclear spectra of NGC 3690/Arp 299B1 (top) and IC 694/Arp 299-A (bottom) together with the Spitzer/IRS spectra.

\section{NUCLEAR REGION OF NGC 3690/Arp 299-B1}

\subsection{Nuclear versus Circumnuclear Spectra}

The GTC/CC nuclear spectrum of NGC 3690 was observed under excellent seeing conditions, $\mathrm{FWHM}_{8.7 \mu \mathrm{m}}=0^{\prime}$ '3. Thus the unresolved MIR emission originates from a region of $\leqslant 60 \mathrm{pc}$ in size. The nuclear spectrum shows dust continuum emission likely produced by the obscured AGN and a moderately deep $9.7 \mu \mathrm{m}$ silicate feature. The apparent depth of the silicate feature, defined as $S_{\mathrm{Si}}=\ln \left(f_{\text {cont }} / f_{\text {feature }}\right)$, is $S_{\mathrm{Si}}=0.8$. There is faint nuclear $11.3 \mu \mathrm{m}$ polycyclic aromatic hydrocarbon (PAH) emission (see Figure 2) with an equivalent width (EW) of $<0.01 \mu \mathrm{m}$.

The $\sim 1 \mathrm{kpc} \times 1 \mathrm{kpc}$ Spitzer $/$ IRS spectrum shows a composite (AGN/SF) nature. It shows 8.6 and $11.3 \mu \mathrm{m}$ PAH features together with a strong continuum due to hot dust (see also Gallais et al. 2004; AAH09). The EW of the $11.3 \mu \mathrm{m}$ PAH feature measured using a local continuum is $\sim 0.05 \mu \mathrm{m}$ (Pereira-Santaella et al. 2010), which is typical of AGN (see Hernán-Caballero \& Hatziminaoglou 2011). This agrees with the presence of the unresolved IR-bright source B1, which has an important hot dust contribution at $\lambda \geqslant 2 \mu \mathrm{m}$ based on the observed near-IR 

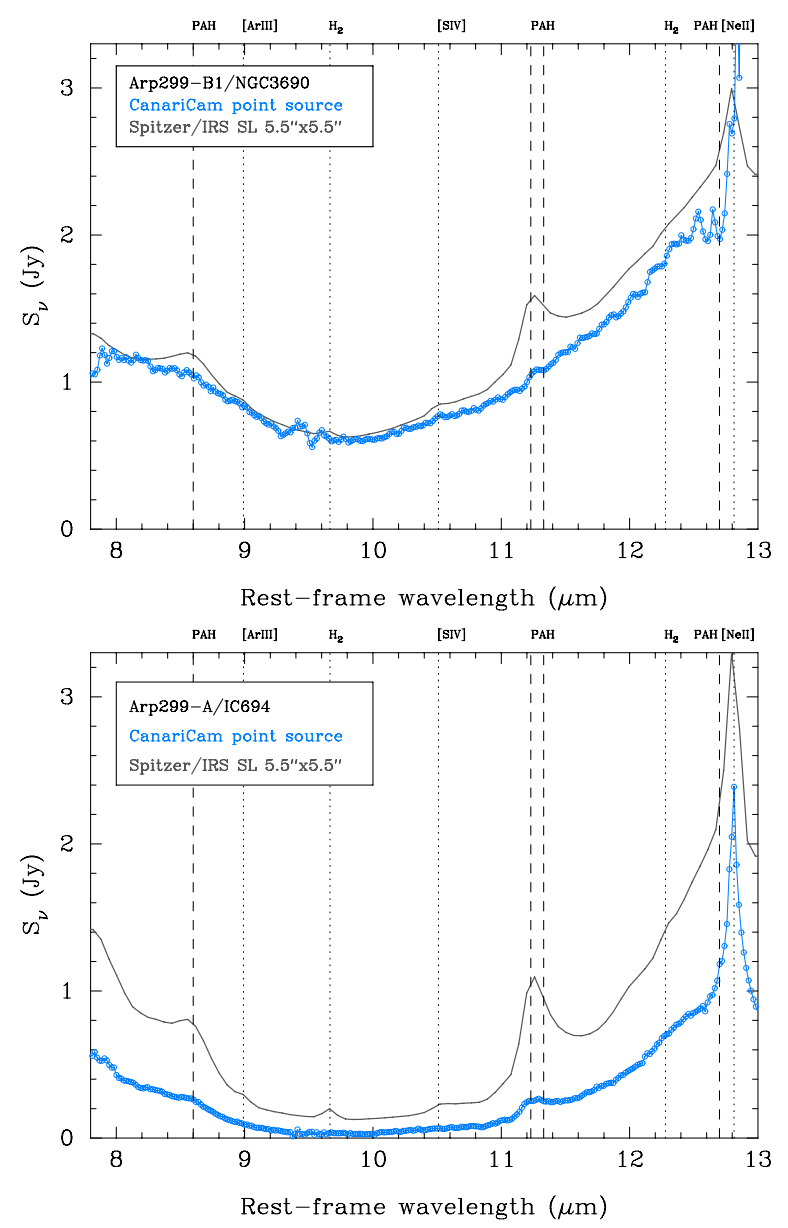

Figure 2. GTC/CC spectra (open symbols) and Spitzer/IRS spectra (black line) of the nuclear regions of Arp 299-B1 (upper panel) and Arp 299-A (lower panel) extracted as point sources. We mark emission lines and PAH features. The noise in the GTC/CC spectra around $9.5 \mu \mathrm{m}$ is due to the $\mathrm{O}_{3}$ telluric band.

(A color version of this figure is available in the online journal.)

CO index, possibly associated with an AGN. On scales of a few arcseconds, $\mathrm{B} 1$ is also surrounded by star clusters and bright $\mathrm{H}$ II regions (AAH00).

\subsection{Modeling with the CLUMPY Torus Models}

Clumpy torus models reproduce satisfactorily the nuclear IR emission of Seyfert galaxies and give an estimate of the AGN bolometric luminosity (Ramos Almeida et al. 2009, 2011; Hönig et al. 2010; Alonso-Herrero et al. 2011). In this section we model the IR spectral energy distribution (SED) and MIR spectrum of NGC 3690 to estimate the AGN's bolometric luminosity.

We constructed the nuclear IR SED of NGC 3690 with the GTC/CC Si-2 $8.7 \mu \mathrm{m}$ point source measurement of $1050 \pm$ $200 \mathrm{mJy}$ and the Hubble Space Telescope/NICMOS $2.2 \mu \mathrm{m}$ non-stellar measurement (AAH00). We included the Keck $1^{\prime \prime}$ diameter 3.2 and $17.9 \mu \mathrm{m}$ (Soifer et al. 2001) and the Kuiper Airborne Observatory $37.7 \mu \mathrm{m} \mathrm{8} 8$.5 beam flux densities (Charmandaris et al. 2002) as upper limit since they are probably contaminated by non-AGN emission. Figure 3 shows the nuclear IR SED.

We used the Nenkova et al. (2008) clumpy torus models, also known as CLUMPY, and the Bayesian fitting routine BAYESCLUMPY (Asensio Ramos \& Ramos Almeida 2009) to fit the nuclear IR emission of NGC 3690. A Bayesian approach allows the proper handling of the intrinsic degeneracies of

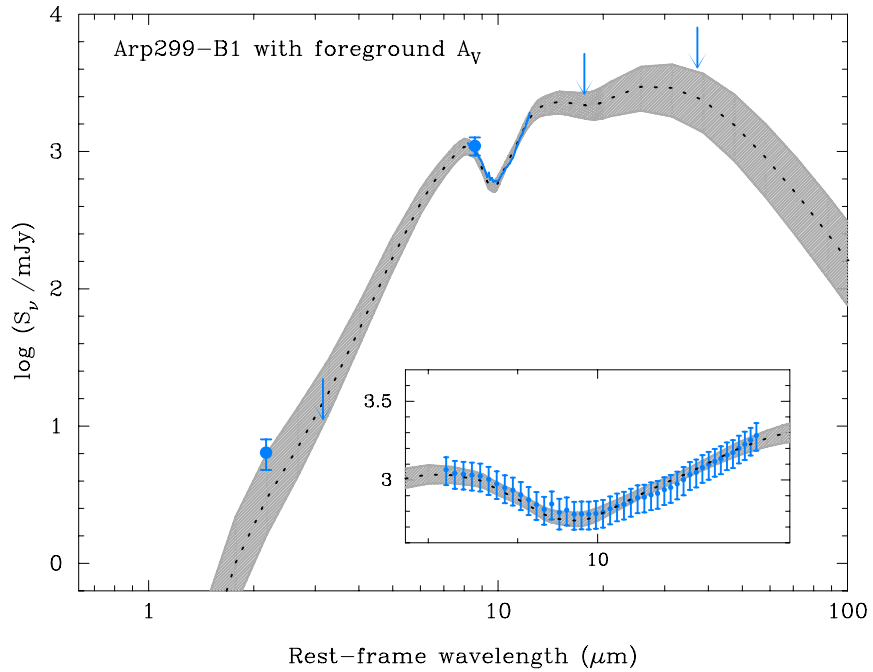

Figure 3. Best median fit using the CLUMPY torus models (black dotted line) and $1 \sigma$ confidence (shaded region) to the nuclear IR SED (filled blue dots and arrows) and MIR GTC/CC spectrum (re-sampled to 45 data points, blue line) of NGC 3690/Arp 299-B1. The inset shows the GTC/CC spectrum (filled blue dots with errors) and best-fit models as above.

(A color version of this figure is available in the online journal.)

Table 1

CLUMPY Torus Models for NGC 3690/Arp 299-B1

\begin{tabular}{lcc}
\hline \hline CLUMPY Torus Parameter & Prior/Range & Fit \\
\hline Torus width, $\sigma_{\text {torus }}$ & $15^{\circ}-70^{\circ}$ & $57_{-8}^{\circ+7}$ \\
Torus radial thickness, $Y$ & $5-100$ & $51_{-30}^{+28}$ \\
No. clouds along equator, $N_{0}$ & $1-15$ & $10_{-2}^{+3}$ \\
Cloud optical depth, $\tau_{V}$ & $5-150$ & $45_{-13}^{+15}$ \\
Cloud radial distribution $r^{-q}, q$ & $0-3$ & $2.7_{-0.3}^{+0.2}$ \\
Viewing angle, $i$ & $60^{\circ}-90^{\circ}$ & $75_{-9}^{\circ+8}$ \\
$A_{V}$ (foreground) & 16 mag \pm 7 & $15 \mathrm{mag}_{-3}^{+3}$ \\
\hline
\end{tabular}

Note. In the CLUMPY models the torus radial thickness is defined as $Y=R_{\mathrm{o}} / R_{\mathrm{d}}$, where $R_{\mathrm{O}}$ is the outer radius and $R_{\mathrm{d}}$ is the inner radius.

the CLUMPY models, provides probability distributions of the fitted parameters, and allows the use of priors for the torus parameters.

The detection of a nuclear water maser in Arp 299-B1 (Tarchi et al. 2011) implies an almost edge-on view of the AGN. We restricted the range of the viewing angles to $i=60^{\circ}-90^{\circ}$ as a prior. Foreground dust is clearly present in the nuclear region of this galaxy (see the optical to near-IR color map in Figure 9 of AAH00). We set the prior for the foreground extinction to a Gaussian distribution centered at 16 mag with a 7 mag width, based on the estimates of AAH00. We use the extinction curve of Chiar \& Tielens (2006). For the rest of the CLUMPY model parameters we used the full range (see Table 1).

The best-fit CLUMPY torus model is shown in Figure 3 and the parameters are listed in Table 1 . The fit is achieved with a wide torus, a highly inclined view, and a large number of clouds along the equatorial direction. This results in a high covering factor of $f_{2} \sim 0.9$ (see Figure 10 in Ramos Almeida et al. 2011). The torus extinction along the line of sight of $A_{V}^{\mathrm{LOS}}$ (torus) $\sim 456 \mathrm{mag}$ dominates over the foreground extinction.

The best-fit model to the nuclear IR emission provides an estimate of the AGN bolometric luminosity of $L_{\mathrm{bol}}(\mathrm{AGN})=$ $3.2 \pm 0.6 \times 10^{44} \mathrm{erg} \mathrm{s}^{-1}$. This is about a factor of 10 higher 
than the $0.5-100 \mathrm{keV}$ intrinsic luminosity derived for this system with BeppoSAX with $N_{\mathrm{H}} \sim 1.9 \times 10^{24} \mathrm{~cm}^{-2}$ (Della Ceca et al. 2002). Alternatively we can use the hard X-ray versus $12 \mu \mathrm{m}$ correlation observed for local Seyfert galaxies (Gandhi et al. 2009; Levenson et al. 2009) and the nuclear $12 \mu \mathrm{m}$ monochromatic luminosity. We predict an intrinsic $L_{2-10 \mathrm{keV}}=4.5 \pm 1.4 \times 10^{43} \mathrm{erg} \mathrm{s}^{-1}$.

The ratio of observed (Ballo et al. 2004; Pereira-Santaella et al. 2011) to predicted intrinsic $2-10 \mathrm{keV}$ luminosities is of the order of 500 , which would only be compatible with the maximum value of $N_{\mathrm{H}} \sim 4 \times 10^{24} \mathrm{~cm}^{-2}$ fitted from the BeppoSAX data (Della Ceca et al. 2002). Both MIR estimates of the AGN luminosity are higher than direct measurements, although the highest possible $N_{\mathrm{H}}$ value would produce compatible luminosities. A possibility would be that the nuclear MIR emission had an important contribution from SF. AAH00 estimated that the SF activity in Arp 299-B1 is approximately five times less than in Arp 299-A. Then using the nuclear $12 \mu \mathrm{m}$ fluxes of Arp 299-A and Arp 299-B1 we predict that SF would only contribute $\sim 15 \%$ of the observed $12 \mu \mathrm{m}$ nuclear emission from Arp 299-B1. We would therefore favor the high $N_{\mathrm{H}}$ value rather than a strong contamination from nuclear SF. Finally, it is possible that the hard X-ray versus MIR relation might not be applicable to all Compton-thick AGN.

\section{NUCLEAR REGION OF IC 694/Arp 299-A}

\subsection{Nuclear versus Circumnuclear Spectra}

The GTC/CC nuclear spectrum of IC 694 (Figure 2) shows clear PAH emission at 8.6 and $11.3 \mu \mathrm{m}$ indicative of the presence of SF on nuclear scales of $\leqslant 120 \mathrm{pc}$. This is consistent with results from high angular resolution near-IR (AAH00) and radio (Pérez-Torres et al. 2009) observations of this nucleus. We measured a GTC/CC nuclear EW $(11.3 \mu \mathrm{mPAH})=$ $0.12 \pm 0.01 \mu \mathrm{m}$, which is lower than that measured from the kpc-scale Spitzer/IRS spectrum $(0.23 \pm 0.01 \mu \mathrm{m}$, see PereiraSantaella et al. 2010). These are typical of galaxies with a composite (AGN/SF) activity (Hernán-Caballero \& Hatziminaoglou 2011). Moreover, the decreased nuclear EW with respect to the $1 \mathrm{kpc}$ scale one also indicates a higher relative contribution in the nuclear region from continuum emission, possibly produced by dust heated by the putative AGN. This is similar to findings for nearby Seyferts with nuclear SF activity (Esquej et al. 2013).

\subsection{Fit to the Nuclear Spectrum}

Deep silicate features, as in the nuclear spectrum of Arp 299-A, cannot be reproduced with the CLUMPY torus models (Levenson et al. 2007; Alonso-Herrero et al. 2011; GonzálezMartín et al. 2013). Instead, Levenson et al. (2007) demonstrated that spherical dusty shell models where the nuclear source is deeply embedded in a smooth distribution of geometrically and optically thick material can produce such deep features. Moreover, the reprocessed emission in these models does not depend on the input spectrum of the heating source. We obtained the best-fit shell model to the GTC/CC spectrum of Arp299-A with a total optical depth through the shell of $\tau_{V}=162$, a maximum temperature at the inside surface of the shell of $T_{\max }=1500 \mathrm{~K}$, $Y=100$ (defined as in Table 1), and an $r^{-1}$ density profile. These are similar to the values obtained for the deep silicate feature observed in the ultraluminous IR galaxy IRAS 08572+3915 (Levenson et al. 2007). While the overall fit to Arp 299-A is reasonable, it fails to reproduce the shape of the silicate feature

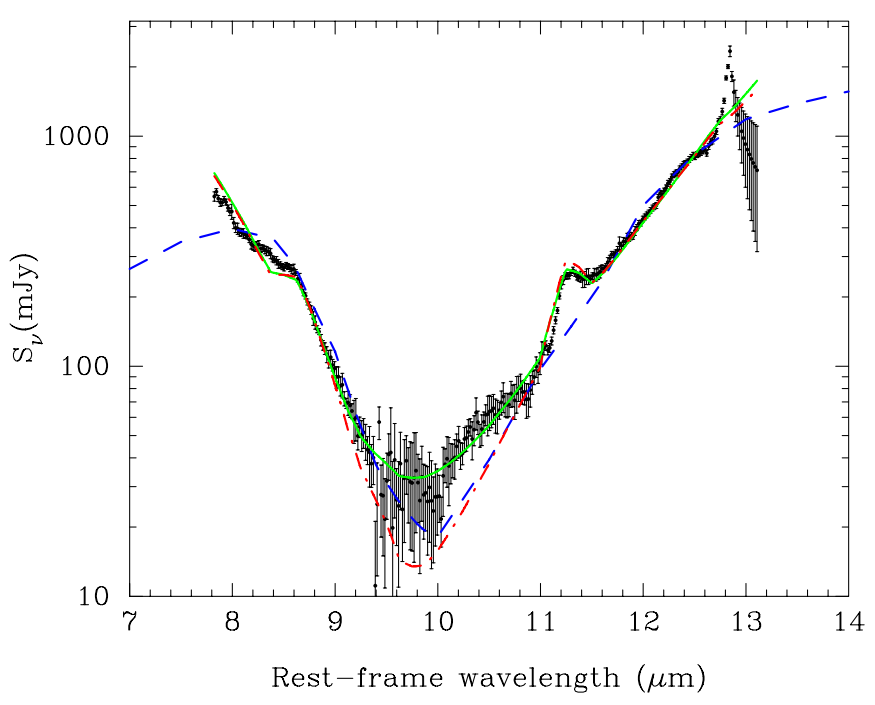

Figure 4. Fit to the GTC/CC nuclear spectrum of IC 694/Arp 299-A. The error bars used for the fit are estimated from the scatter of the points in narrow wavelength bins. The blue dashed line is the best fit with the spherical dusty shell models of Levenson et al. (2007). The dot-dashed red line is the fit using a blackbody + the Orion bar PAH spectrum with silicate absorption as modeled with the Trapezium silicate grains following Roche et al. (2007). The solid green line includes additionally a power-law component that fills in the $\sim 9-11 \mu \mathrm{m}$ spectral range. The error bars around the $[\mathrm{Ne}$ II $] 12.81 \mu \mathrm{m}$ line were increased artificially to exclude it from the fit.

(A color version of this figure is available in the online journal.)

around $10-11 \mu \mathrm{m}$ (see Figure 4). Also, the dusty shell model does not fit the strong nuclear PAH emission, as it does not include a SF component.

Alternatively, we fitted the GTC/CC spectrum with a combination of emission and absorption components using the technique described by Roche et al. (2007). We first tried a blackbody + the Orion bar PAH spectrum suffering silicate absorption. This fit is shown as a dashed line in Figure 4 and has $\chi^{2} / N=5.9$, where $N$ is the number of degrees of freedom. The silicate optical depth is $\tau_{9.7 \mu \mathrm{m}}=1.4\left(A_{V}=21 \mathrm{mag}\right.$, assuming a dust screen geometry) with the Trapezium silicate grain profile, which is representative of those of molecular clouds. Using the curve of the $\mu$ Cephei supergiant gave a poorer fit, but these silicate grains seem to be more appropriate for sources with extremely deep silicate features (Roche et al. 2007).

Although these two components provide quite a good qualitative agreement over most of the spectrum, the fit falls below the data points between 9 and $11 \mu \mathrm{m}$, as found with the dusty shell model fit above. To improve the fit, we added an IR power-law component ( $f_{v} \propto v^{-2}$, see Ramos Almeida et al. 2009). This fills in the 9-11 $\mu \mathrm{m}$ spectrum (Figure 4) and improves the fit $\left(\chi^{2} / N=4.1\right)$. The derived silicate optical depth is $\tau_{9.7 \mu \mathrm{m}}=1.6$ or $A_{V}=24 \mathrm{mag}$, in agreement with other IR estimates (Gallais et al. 2004; AAH00; AAH09).

The power-law component contributes approximately $35 \%$ of the observed $9 \mu \mathrm{m}$ flux density in the nuclear GTC/CC spectrum of IC 694. We propose that this emitting component might be associated with dust heated by the putative AGN and also be responsible for the decreased nuclear EW (11.3 $\mu \mathrm{m}$ PAH). From the power-law monochromatic $12 \mu \mathrm{m}$ luminosity we estimated an intrinsic $L_{2-10 \mathrm{keV}} \sim 1 \pm 0.2 \times 10^{43} \mathrm{erg} \mathrm{s}^{-1}$ using the Gandhi et al. (2009) relation, as in Section 3.2. The putative AGN in IC 694 would be about five times less luminous than that in NGC 3690. 


\section{DISCUSSION AND CONCLUSIONS}

We obtained GTC/CC high angular resolution $\left(0.3-00^{\prime \prime} 6\right.$, $\sim 60-120$ pc) MIR spectra of the interacting galaxy Arp 299. We presented evidence of deeply embedded AGN activity in both nuclei thanks to the improved angular resolution of these data of almost a factor of 10 with respect to previous MIR spectroscopy of this system.

The GTC/CC nuclear spectrum of NGC 3690/Arp 299-B1 shows a strong dust continuum with a moderate silicate feature in absorption. The nuclear $11.3 \mu \mathrm{m}$ PAH feature is weak compared with that in the $1 \mathrm{kpc}$ scale Spitzer/IRS spectrum. We fitted the IR emission of NGC 3690 with the CLUMPY models. The dust is located in a high covering factor torus and heated by an AGN with $L_{\text {bol }}(\mathrm{AGN})=3.2 \pm 0.6 \times 10^{44} \mathrm{erg} \mathrm{s}^{-1}$. The torus high extinction along the line of sight dominates over the foreground extinction.

The GTC/CC nuclear (region $\leqslant 120 \mathrm{pc}$ in size) spectrum of IC 694/Arp 299-A shows a very deep silicate feature reflecting the highly embedded nature of this source with a fitted $\tau_{9.7 \mu \mathrm{m}}=1.6\left(A_{V}=24 \mathrm{mag}\right.$, for a dust screen geometry). There is strong nuclear SF based on the detection of the $8.6 \mu \mathrm{m}$ and $11.3 \mu \mathrm{m}$ PAH features. The lower EW of the nuclear $11.3 \mu \mathrm{m}$ PAH feature compared with the kpc-scale IRS value suggests the presence of a MIR continuum that also fills in the silicate feature within $\sim 9-11 \mu \mathrm{m}$. If this nuclear continuum emitting component is due to dust heated by an AGN, then we estimated an AGN luminosity about five times less than that in NGC 3690.

The interaction of the Arp 299 system started at least $750 \mathrm{Myr}$ ago (Hibbard \& Yun 1999) but the system has not yet fully merged. The projected nuclear separation is $\sim 4.5 \mathrm{kpc}$ and the system is experiencing intense SF spread out over several kiloparsecs (Charmandaris et al. 2002, AAH09). Our work provided evidence of dual dust enshrouded AGN, probably indicative of an early phase of AGN activity. The combined luminosities of the two AGN only account for $\sim 15 \%$ of the total IR luminosity of the system. This relatively small AGN contribution is typical of local LIRGs (see Petric et al. 2011; Alonso-Herrero et al. 2012).

Numerical simulations predict that interaction-induced AGN activity is common. However, dual AGN activity with $L_{\text {bol }}(\mathrm{AGN})>10^{44} \mathrm{erg} \mathrm{s}^{-1}$ would only occur simultaneously for approximately $10 \%$ of the duration of the interaction (see, e.g., van Wassenhove et al. 2012). Therefore, Arp 299 represents an interesting case study to test theoretical predictions for dual AGN activity during the early stages of galaxy interactions.

We are extremely grateful to the GTC staff for their constant and enthusiastic support. We also thank an anonymous referee for comments that helped improve the Letter. The following Spanish Plan Nacional de Astronomía y Astrofísica grants are acknowledged: AYA2009-05705-E (A.A.H., P.E., C.R.A., and M.P.S.), AYA2010-21887-C04 (C.R.A. and J.M.R.E.), AYA2010-18029 (A.A.R.), AYA2010-21161-C0201 (L.C.), and AYA2012-39168-C03-01 (J.M.R.E. and O.G.M.). A.A.R. also acknowledges financial support through the Ramón y Cajal fellowships and Consolider-Ingenio 2010 CSD200900038. C.P. and C.M.T. acknowledge support from NSF grants 0904421 and AST-903672, respectively. N.A.L. and R.M. are supported by the Gemini Observatory, which is operated by the Association of Universities for Research in Astronomy, Inc., on behalf of the international Gemini partnership of Argentina, Australia, Brazil, Canada, Chile, and the United States of America.

Based on observations made with the GTC, installed in the Spanish Observatorio del Roque de los Muchachos of the Instituto de Astrofísica de Canarias, in the island of La Palma. Based party on observations obtained with the Spitzer Space Observatory, which is operated by JPL, Caltech, under NASA contract 1407. This research has made use of the NASA/ IPAC Extragalactic Database (NED) which is operated by JPL, Caltech, under contract with the National Aeronautics and Space Administration.

\section{REFERENCES}

Alonso-Herrero, A., Pereira-Santaella, M., Rieke, G. H., \& Rigopoulou, D. 2012, ApJ, 744, 2

Alonso-Herrero, A., Ramos Almeida, C., Mason, R., et al. 2011, ApJ, 736, 82

Alonso-Herrero, A., Rieke, G. H., Colina, L., et al. 2009, ApJ, 697, 660 (AAH09)

Alonso-Herrero, A., Rieke, G. H., Rieke, M. J., \& Scoville, N. Z. 2000, ApJ, 532, 845 (AAHO0)

Asensio Ramos, A., \& Ramos Almeida, C. 2009, ApJ, 696, 2075

Ballo, L., Braito, V., Della Ceca, R., et al. 2004, ApJ, 600, 634

Charmandaris, V., Stacey, G. J., \& Gull, G. 2002, ApJ, 571, 282

Chiar, J. E., \& Tielens, A. G. G. M. 2006, ApJ, 637, 774

Della Ceca, R., Ballo, L., Tavecchio, F., et al. 2002, ApJL, 581, L9

Dudley, C. C. 1999, MNRAS, 307, 553

Esquej, P., Alonso-Herrero, A., González-Martín, O., et al. 2013, ApJ, in press (arXiv:1311.0703)

Gallais, P., Charmandaris, V., Le Floc'h, E., et al. 2004, A\&A, 414, 845

Gandhi, P., Horst, H., Smette, A., et al. 2009, A\&A, 502, 457

García-Marín, M., Colina, L., Arribas, S., Alonso-Herrero, A., \& Mediavilla, E. 2006, ApJ, 650, 850

Gehrz, R. D., Sramek, R. A., \& Weedman, D. W. 1983, ApJ, 267, 551

González-Martín, O., Masegosa, J., Márquez, I., \& Guainazzi, M. 2009, ApJ, 704, 1570

González-Martín, O., Rodríguez Espinosa, J. M., Díaz-Santos, T., et al. 2013, A\&A, 553, A35

Hernán-Caballero, A., \& Hatziminaoglou, E. 2011, MNRAS, 414, 500

Hibbard, J. E., \& Yun, M. S. 1999, AJ, 118, 162

Hönig, S., Kishimoto, M., Gandhi, P., et al. 2010, A\&A, 515, A23

Houck, J. R., Roellig, T. L., van Cleve, J., et al. 2004, ApJS, 154, 18

Levenson, N. A., Radomski, J. T., Packham, C., et al. 2009, ApJ, 703, 390

Levenson, N. A., Sirocky, M. M., Hao, L., et al. 2007, ApJL, 645, L45

Neff, S., Ulvestad, J. S., \& Teng, S. H. 2004, ApJ, 611, 186

Nenkova, M., Sirocky, M. M., Nikutta, R., Ivezić, Z., \& Elitzur, M. 2008, ApJ, 685,160

Pereira-Santaella, M., Alonso-Herrero, A., Rieke, G. H., et al. 2010, ApJS, 188,447

Pereira-Santaella, M., Alonso-Herrero, A., Santos-Lleo, M., et al. 2011, A\&A, 535, A93

Pérez-Torres, M. A., Alberdi, A., Romero-Cañizares, C., \& Bondi, M. 2010, A\&A, 519, L5

Pérez-Torres, M. A., Romero-Cañizares, C., Alberdi, A., \& Polatidis, A. 2009, A\&A, 507, L17

Petric, A. O., Armus, L., Howell, J., et al. 2011, ApJ, 730, 28

Ramos Almeida, C., Levenson, N. A., Alonso-Herrero, A., et al. 2011, ApJ, 731, 92

Ramos Almeida, C., Levenson, N. A., Rodríguez Espinosa, J. M., et al. 2009, ApJ, 702, 1127

Rieke, G. H., \& Low, F. J. 1972, ApJL, 176, L95

Roche, P. F., Packham, C., Aitken, D. K., \& Mason, R. 2007, MNRAS, 375,99

Smith, J. D. T., Armus, L., Dale, D. A., et al. 2007, PASP, 119, 1133

Soifer, B. T., Neugebauer, G., Matthews, K., et al. 2001, AJ, 121, 1213

Tarchi, A., Castangia, P., Henkel, C., Surcis, G., \& Menten, K. M. 2011, A\&A, 525,91

Telesco, C. M., Ciardi, D., French, J., et al. 2003, Proc. SPIE, 4841, 913

Telesco, C. M., Decher, R., \& Gatley, I. 1985, ApJ, 299, 896

van Wassenhove, S., Volonteri, M., Mayer, L., et al. 2012, ApJL, 748, L17 